\title{
Heart failure - does ejection fraction hold any relevance?
}

\author{
Om Prakash Yadava ${ }^{1} \mathbb{D}$
}

Published online: 13 November 2021

(c) Indian Association of Cardiovascular-Thoracic Surgeons 2021

The recent revelation of the potential of two new groups of drugs viz. angiotensin receptor neprilysin inhibitors (ARNI) and sodium glucose transporter-2 (SGLT-2) inhibitors, with their repertoire extending beyond the thresholds of left ventricular ejection fraction (LVEF), has raised a new conundrum - is LVEF a holy grail in the management of heart failure (HF). The EMPEROR-Preserved trial [1] of SGLT-2 inhibitors, presented recently at the European Cardiology Congress, met its composite primary end-point, even in patients with normal ejection fraction (EF), thus mirroring its role in patients of $\mathrm{HF}$ with impaired $\mathrm{EF}$, as was demonstrated in the EMPEROR-Reduced trial [2]. This has led to a view point that even though LVEF may be an important parameter to consider for device therapy, it has very little relevance for choosing drugs for medical management of HF.

Even in a surgical cohort, HF was found to be a more important and valid prognosticator for outcomes than EF. In a population-based retrospective cohort study from Canada looking at 40,083 patients undergoing Coronary Artery Bypass Graft (CABG) surgery, Sun et al. [3] found 30-day mortality to be higher at $3.3 \%$ in patients with $\mathrm{HF}$, despite preserved $\mathrm{EF}$, as compared to reduced EF but no HF (1.3\%). After adjustment of all confounders, the hazard ratio (HR) for reduced $\mathrm{EF}$ without $\mathrm{HF}$, preserved $\mathrm{EF}$ with $\mathrm{HF}(\mathrm{HFpEF})$ and reduced $\mathrm{EF}$ with $\mathrm{HF}$ (HFrEF) were 1.56 (95\% CI, 1.23-1.97); 2.60 (95 CI, 1.98-3.40) and 3.92 (95\%, CI, 3.15-4.90), respectively, compared with those with no HF and preserved EF (ref. Fig. 1). This prognostic value of $\mathrm{HF}$ was reflected in the long-term results too. In patients with preserved $\mathrm{EF}$, heart failure portended a three-fold higher mortality than without HF (ref. Fig. 2).

The same message emanates from the SWEDEHEART registry [4], where HF was found to be an independent predictor of both short- and long-term mortality following CABG, irrespective of EF. The adjusted HRs for 30-day mortality were $2.25,1.83$ and 2.52 in patients with no HF

Om Prakash Yadava

op_yadava@yahoo.com

1 National Heart Institute, New Delhi, India and reduced $\mathrm{EF}, \mathrm{HFpEF}$ and $\mathrm{HFrEF}$ respectively. At a mean follow-up of 6 years, corresponding HRs were 1.47, 1.62 and 2.29 , respectively, suggesting that even though EF was a predictor of 30-day mortality, it was HF which was a more robust predictor of late mortality. Thus, more attention should be paid to the presence and management of HF, rather than being 'EF-centric' [3].

There are essentially four groups of drugs which are the pillars of HF management as on date and have been shown to have survival benefits. These are beta blockers, mineralocorticoid receptor antagonists (MRAs), ARNIs and SGLT-2 inhibitors. They all seem to act across the entire continuum of the spectrum of HF and their therapeutic effects go beyond the conventionally created three thresholds of LVEF-HFrEF, heart failure with mid-range EF (HFmrEF) and HFpEF. Even if one looks at the pathophysiology of HF, it is not just the mechanistic LVEF which contributes to HF, but there are also elements of neuro-hormonal activation, renal function and other non-hormonal factors, which come into play. Because most of these drugs have pharmacological actions on a panoply of patho-physiological mechanisms of $\mathrm{HF}$, including direct effects on renal function, it adds credence to the view that one has to go beyond the silo-based thinking of just $\mathrm{EF}$ for categorisation of HF patients.

Furthermore, the measurement of LVEF is prone to experimental errors and may vary from time to time, depending on the clinical situation and other parameters like congestion, preload and afterload. LVEF does not even represent disease phenotypes accurately and there is a lot of blurring and overlaps at the boundary zones between HFpEF, HFmrEF and HFrEF. Pharmacotherapy with the four basic groups for HF treatment cuts across these arbitrary divisions, as also underscored in a panel discussion during the recent Heart Failure Society of the America 2021 Annual Scientific meeting.

Proponents of relevance of LVEF in medical management of HF still maintain that the physiology in HFrEF and HFpEF is different, and that the drugs have a higher efficacy as the LVEF goes down and that the efficacy wanes as the EF rises. Therefore, usefulness of beta blockers in HF 
Fig. 1 Estimated 30-day survival after isolated coronary artery bypass grafting surgery by heart failure status (HF) and ejection fraction. HFpEF indicates HF with preserved ejection fraction; HFrEF, HF with reduced ejection fraction; $\mathrm{pEF}$, preserved ejection fraction; rEF, reduced ejection fraction Commons Licence) [3] (reproduced under Creative
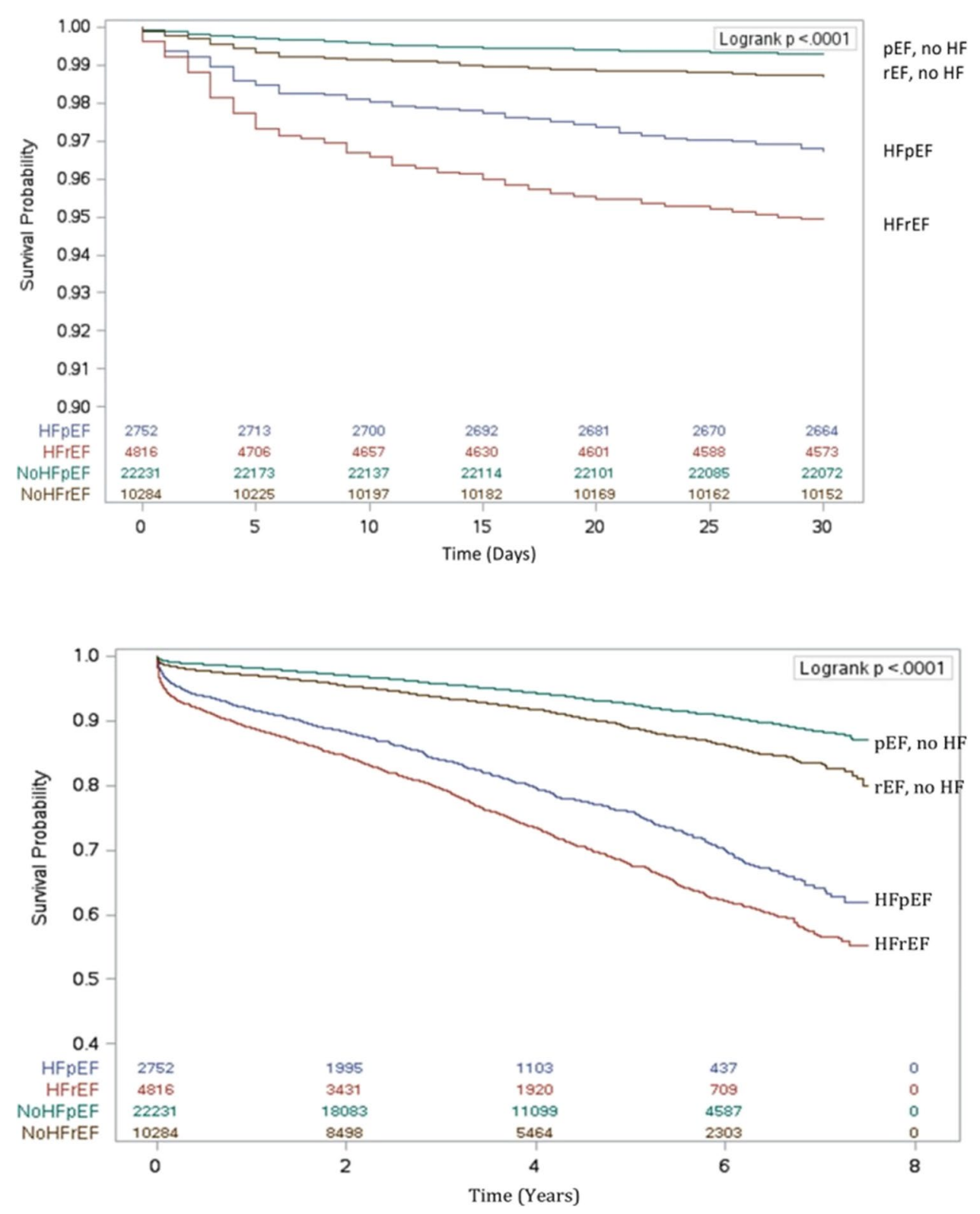

Fig. 2 Estimated long-term survival after isolated coronary by heart failure (HF) status and ejection fraction. HFpEF indicates HF with preserved ejection fraction; HFrEF, HF with reduced ejection fraction; $\mathrm{pEF}$, preserved ejection fraction; rEF, reduced ejection fraction [3] (reproduced under Creative Commons Licence) artery bypass grafting surgery

patients with preserved EF has been challenged. However, as Randall C. Starling from the Cleveland Clinic pointed out [5], the patients with coronary artery disease, systemic hypertension and atrial fibrillation may benefit from the use of beta blockers, even if they have normal LVEF. He does not think LVEF as a guide to HF therapy 'is ever going to be eliminated, but I think more and more, we are going to see treatments that will not be restricted based on a specific ejection fraction. The emergence of HF medicines that are agnostic to the ejection fraction, I think is a great thing. And we may windup seeing more patients treated' [6]. All these four classes are LVEF agnostic and probably benefits extend to the entire range of EF, including up to LVEF of $50-55 \%$.

\section{Prescribing inertia}

This also brings us to another conclusion in response to the conundrum of sequencing of drugs. Traditionally it has been taught that one can introduce HF drugs sequentially and in incrementally larger doses, and this at times took months to reach an optimum level. Quite often the time delay to get clinical benefits led the patients themselves, or the treating physician, losing sight of the trends, so that the treatment regimens were either aborted or never reached an optimum level. This so-called prescribing inertia, as also the findings that even as short as 30 days of quadruple therapy of the four foundational therapeutic regimens led to a reduction in mortality by more than three-quarters, led 
Fig. 3 Drug, interventional and device treatment for heart failure with reduced ejection fraction (HFrEF). ACE-I, angiotensin-converting enzyme inhibitor; Afib, atrial fibrillation; ARB, angiotensin receptor blocker; ARNI, angiotensin receptor/neprilysin inhibitor; CRT, cardiac resynchronisation therapy; HTX, heart transplantation; LBBB, left bundle branch block; LVAD, left ventricular assist device; MR, mitral regurgitation; MRA, mineralocorticoid receptor antagonist; PVI, pulmonary vein isolation; SGLT-2, sodiumglucose co-transporter 2; SR, sinus rhythm; TSAT, transferrin saturation [7] (reproduced with permission)

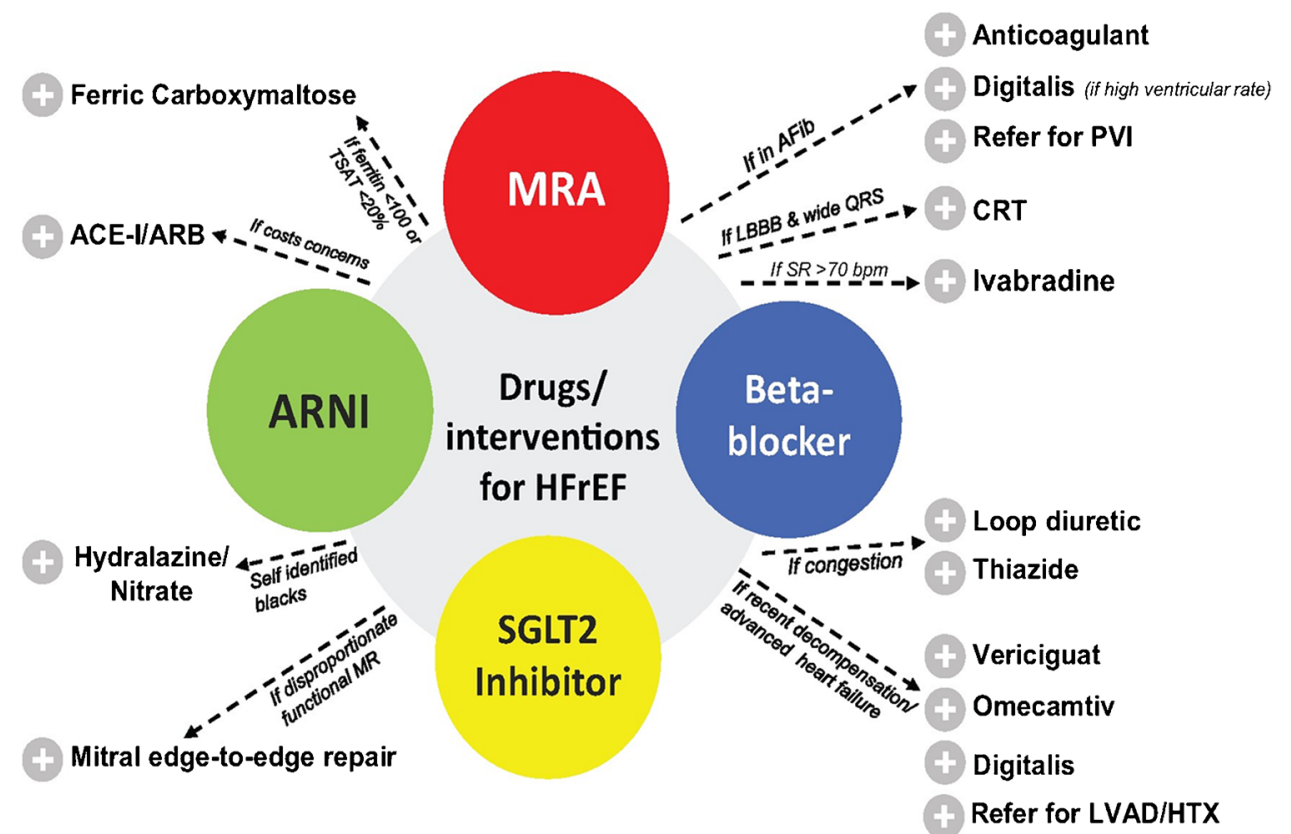

Dr. Fonarow, speaking at the 2020 Virtual annual meeting of the Heart Failure Society of America, advocating introduction of all four classes of drugs simultaneously on day one. He further stressed, 'the benefits from each of the four classes involve distinct physiologic pathways and hence are not diminished by concurrent treatment ......... Overcome inertia by prescribing all four drug classes at the time of diagnosis'. Though Dr. Fonarow's exhortation, and the latest European guideline's [6] class I recommendation for the use of drug classes is limited to HFrEF, now with the EMPEROR-Preserved findings firmly entrenched, probably this statement would extend to HFpEF also, thereby making the rigid categorisation of HF according to LVEF redundant.

Changing gears, a host of other mechanistic pathways in $\mathrm{HF}$ are being evaluated for drug development, and the times are exciting with newer molecules seeing the light of the day. Soluble guanylate cyclase stimulator vericiguat is already approved in the USA, and another-omecamtiv mecarbil-is in the pipe line and likely to get the nod soon. For the moment, the message is very clear that all patients with HF, irrespective of LVEF, should be treated early and with all 4 basic foundational groups of drugs. As against the beta blockers and ARNIs, which may need gradual uptitration, SGLT-2 inhibitors do not need any up-titration and therefore are easy to prescribe and one can hit the deck running for the management of HF. Even patients who currently have no symptoms should be quickly addressed and all four groups of drugs should be brought on board with expediency. Thereafter, tweaking with other modalities of treatment of HF, including devices and surgery, can be undertaken depending on the clinical requirements of an individual patient in a customised and personalised manner (ref. Fig. 3) [7].

Pre-eminence of $\mathrm{HF}$ as a prognosticator has lately dawned on the medical fraternity, and HF treatment is indeed witnessing a paradigm shift, with 'light at the end of the tunnel' a reality, rather than a wishful figment of imagination.

\section{Declarations}

Informed consent Not required.

Conflict of interest The author declares no competing interests.

\section{References}

1. Anker SD, Butler J, Filippatos G, et al. Empagliflozin in heart failure with a preserved ejection fraction. N Engl J Med. 2021. https://doi.org/10.1056/NEJMoa2107038.

2. Packer M, Anker SD, Butler J, et al. Cardiovascular and renal outcomes for empagliflozin in heart failure. $\mathrm{N}$ Engl $\mathrm{J}$ Med. 2020;383:1413-24.

3. Sun LY, Tu JV, Bader Eddeen A, Liu PP. Prevalence and long-term survival after coronary artery bypass grafting in women and men with heart failure and preserved versus reduced ejection fraction. $\mathrm{J}$ Am Heart Assoc. 2018;7:e008902. https://doi.org/10.1161/JAHA. 118.008902.

4. Dalén M, Lund LH, Ivert T, Holzmann MJ, Sartipy U. Survival after coronary artery bypass grafting in patients with preoperative heart failure and preserved vs reduced ejection fraction. JAMA Cardiol. 2016;1:530-8. https://doi.org/10.1001/jamacardio.2016.1465. 
5. McDonagh TA, Metra M, Adamo M, et al. 2021 ESC Guidelines for the diagnosis and treatment of acute and chronic heart failure. Eur Heart J. 2021;42:3599-726.

6. Stiles S. Ejection fraction for guiding HF therapy: forget about it? Medscape - Sept 29, 2021.
7. Bauersachs J. Heart failure drug treatment: the fantastic four. Eur Heart J. 2021;42:681-3.

Publisher's note Springer Nature remains neutral with regard to jurisdictional claims in published maps and institutional affiliations. 